\title{
Reducing pain and opioid consumption after body contouring of the breast by application of a perioperative nerve block: a systematic review
}

\author{
Derek B. Asserson ${ }^{1}$, David E. Sahar ${ }^{2}$ \\ ${ }^{1}$ California Northstate University College of Medicine, Elk Grove, CA; ${ }^{2}$ Division of Plastic Surgery, Department of Surgery, University of \\ California Davis Medical Center, Sacramento, CA, USA
}

\begin{abstract}
Background Pain in the postoperative body contouring patient has traditionally been managed with narcotic medication. In an effort to minimize side effects and prevent addiction, plastic surgeons are searching for novel ways to provide adequate analgesia, one of which is nerve blocks. This study was conducted with a meta-analysis that evaluates the efficacy of these blocks for patients who undergo breast surgery.

Methods A search of the PubMed/MEDLINE database for articles including the terms "postoperative analgesia" OR "postoperative pain management" AND "in plastic surgery" OR "in cosmetic surgery" OR "in elective surgery" in February 2019 generated five studies on elective breast augmentation and reduction mammoplasty that reported pain scores and quantities of opioids consumed. Independent samples t-tests, one-way analysis of variance, and a random effects model were implemented for evaluation.
\end{abstract}

Results A total of 317 patients were identified as having undergone body contouring of the breast, about half of which received a nerve block. Pain scores on a 1-10 scale and opioid dose-equivalents were calculated. Those who were blocked had an average score of 2.40 compared to 3.64 for those who did not $(P<0.001)$, and required an average of 5.20 less narcotic doses $(P<0.001)$. Pain relief following subpectoral augmentation was best achieved with type-II blocks as opposed to type-I and type-II with serratus plane $(P<0.001)$.

Conclusions The opioid epidemic has extended to all surgical specialties. Implementation of a nerve block seems to be an efficacious and cost-effective mechanism to not only help with postoperative pain, but also lower the need for narcotics, especially in subpectoral augmentation.

Keywords Opioids / Pain / Body contouring / Breast / Nerve block
Correspondence: Derek B. Asserson California Northstate University College of Medicine, 9700 West Taron Drive, Elk Grove, CA 95757, USA

Tel: +1-916-686-7300

Fax: +1-916-686-8140

E-mail: derek.asserson@gmail.com

\section{INTRODUCTION}

The "Opioid Crisis" began in the late 1990s when pharmaceutical companies reassured physicians that patients would achieve adequate pain control without becoming addicted to narcotic medication [1]. Synthetic drugs soon came around, and the overdose death tally of all opioids, legal and illegal, rose from 3 million Americans in 1999 to 15 million in 2017, with a quoted misuse rate of $21 \%-29 \%$ and disorder rate of $8 \%-12 \%$ [1].

Blame is frequently placed on surgeons, who have lacked edu- 
cation on pain management in their training curricula [2]. As a result, up to $71 \%$ of all prescribed opioids go unused, and these leftovers may get into the hands of patients and non-patients who do not require such strong analgesia [3]. Abuse has previously been commented on in the obstetric [4], orthopedic [5], and otolaryngological [6] literature. Plastic surgeons, in particular, have been found to prescribe almost double the amount of opioids that patients who undergo outpatient procedures actually consume [7].

Nerve blocks of regional muscle groups are becoming a more common way to provide comfort to surgical patients who require day management beyond intravenous sedation and local anesthesia, especially when it comes to elective body contouring, a sector of plastic surgery that is notorious for its prescription of narcotics. However, administration of nerve blocks is not standard practice [8] and the specifics of nerve block techniques, namely where to target the muscle in subpectoral breast augmentation [9], are not yet fully agreed on.

With national attention on the epidemic, the plastic surgery community must do its part to help ameliorate the problem. We looked to the current literature to identify ways in which plastic surgeons minimize their opioid prescriptions while also reducing pain in the postoperative setting to ensure comfort and few side effects to patients who undergo elective breast cases. Within this systematic review, we present a meta-analysis of the effectiveness of such nerve block techniques.

\section{METHODS}

We searched the PubMed/MEDLINE database for articles containing the terms "postoperative analgesia" OR "postoperative pain management" AND "in plastic surgery" OR "in cosmetic surgery" OR "in elective surgery" in February 2019. After title screening, we applied inclusion criteria to eliminate unwarranted articles. For the purpose of this study, body contouring of the breast included breast augmentation and reduction mammoplasty. Both retrospective and prospective reviews were included. Articles were required to have complete data on type of analgesic intervention: when the anesthetic was given, how the anesthetic was given, where the anesthetic was given, and what anesthetic was given. They were also required to report on at least one of pain scores and quantities of opioid doses. Because of great variation in available pain scales (numeric rating scale [NRS], visual analog scale [VAS], etc.), there was no requirement of type implemented to be included in the meta-analysis. Doses of narcotics were converted to equivalents using standard adult dosages provided by the online resource Medscape.

\section{Pectoral nerve blocks}

Type-I pectoral blocks are devised to anesthetize the medial and lateral pectoral nerves, which innervate the pectoralis major and pectoralis minor, under ultrasound guidance. Type-II pectoral nerve blocks start with a type-I block and are followed by an injection between the pectoralis minor and serratus anterior to establish a blockade of the upper intercostal nerves. Type-II blocks can additionally be followed with an injection between the serratus anterior and latissimus dorsi in order to establish a blockade of thoracic nerves.

\section{Statistical analysis}

Independent samples t-tests were used to evaluate pain scores and dose-equivalents of opioids between groups receiving and not receiving interventions. Pooled variance was calculated when combining studies. For studies that did not report variance, Ma's method of replacing missing variances was utilized, which imputes weighted variance averages observed in other studies [10]. One-way analysis of variance and post hoc testing with Tukey's multiple comparisons test was used to evaluate pain scores between breast augmentation cases with varying pectoral block techniques. A random effects model was incorporated when considering mean difference of dose-equivalents. A P-value less than 0.05 was considered statistically significant. Analyses were performed with JASP version 0.8.6 (Amsterdam, Netherlands).

\section{RESULTS}

The initial database search returned 148 results, which was cut down to 73 after screening by title and five after inclusion criteria were applied.

These studies reported on 317 patients, of which 173 received a nerve block (54.6\%) and 144 did not (45.4\%). All patients were female of similar age, body mass index, and American Society of Anesthesiologists classification, in addition to having undergone similar operation times, for each study. Moreover, four of the studies involved cases of breast augmentation and one of reduction mammoplasty; three utilized preoperative nerve blocks and two utilized postoperative blocks; the erector spinae muscles were blocked in one, intercostals in one, and pectorals in three; bupivacaine was the anesthetic in all instances; and the NRS (0-10 scale) was used in three versus the VAS (0-10 scale) in two (Table 1) [11-15]. Level of evidence varied between II and IV.

Combining all studies, the nerve block group averaged a pain score of $2.40 \pm 1.12$ after 24 hours, whereas the non-nerve block group averaged $3.64 \pm 1.13(\mathrm{P}<0.001)$ (Table 2$)$. The nerve 
Table 1. Analgesic details by paper

\begin{tabular}{|c|c|c|c|c|c|}
\hline Author (year) & Surgery & Nerve block time & Nerve block location & Anesthetic & Pain scale \\
\hline Ekinci et al. (2019) [13] & Augmentation & Postoperative & Pectorala $^{(a)}$ & Bupivacaine & VAS \\
\hline Hidalgo and Pusic (2005) [11] & Augmentation & Preoperative & Intercostal & Bupivacaine & VAS \\
\hline Karaca et al. (2019) [14] & Augmentation & Postoperative & Pectoral ${ }^{(b)}$ & Bupivacaine & VAS \\
\hline Oksuz et al. (2018) [12] & Reduction & Preoperative & Erector spinae & Bupivacaine & NRS \\
\hline Schuitemaker et al. (2019) [15] & Augmentation & Preoperative & Pectoral( ${ }^{()}$ & Bupivacaine & NRS \\
\hline
\end{tabular}

VAS, visual analog scale; NRS, numeric rating scale.

a)Type-I; b'Type-II; ' ${ }^{\text {II }}$ Type-II with serratus plane.

Table 2. Post-surgical pain scores by hour

\begin{tabular}{|c|c|c|c|c|c|c|c|c|c|}
\hline \multirow{2}{*}{ Hours } & \multicolumn{3}{|c|}{ All cases } & \multicolumn{3}{|c|}{ Breast augmentation } & \multicolumn{3}{|c|}{ Reduction mammoplasty } \\
\hline & Nerve block & No nerve block & P-value & Nerve block & No nerve block & P-value & Nerve block & No nerve block & P-value \\
\hline 1 & $3.77 \pm 1.44$ & $6.09 \pm 1.09$ & $<0.001$ & $4.01 \pm 1.53$ & $6.57 \pm 1.22$ & $<0.001$ & $2.19 \pm 0.92$ & $4.27 \pm 0.63$ & $<0.001$ \\
\hline 2 & $2.57 \pm 1.59$ & $4.01 \pm 1.03$ & $<0.001$ & $2.80 \pm 1.74$ & $5.28 \pm 1.12$ & $<0.001$ & $1.61 \pm 0.58$ & $3.59 \pm 0.73$ & $<0.001$ \\
\hline $3-4$ & $2.65 \pm 1.42$ & $4.54 \pm 0.95$ & $<0.001$ & $2.85 \pm 1.56$ & $4.69 \pm 1.06$ & $<0.001$ & $1.33 \pm 0.48$ & $3.18 \pm 0.58$ & $<0.001$ \\
\hline $6-8$ & $2.61 \pm 1.20$ & $4.14 \pm 0.90$ & $<0.001$ & $2.82 \pm 1.32$ & $4.41 \pm 0.99$ & $<0.001$ & $1.23 \pm 0.43$ & $2.81 \pm 0.58$ & $<0.001$ \\
\hline $12-16$ & $1.26 \pm 0.91$ & $3.21 \pm 0.83$ & $<0.001$ & $1.30 \pm 1.01$ & $3.55 \pm 0.88$ & $<0.001$ & $1.09 \pm 0.03$ & $2.31 \pm 0.64$ & $<0.001$ \\
\hline 24 & $2.40 \pm 1.12$ & $3.64 \pm 1.13$ & $<0.001$ & $2.60 \pm 1.21$ & $3.92 \pm 1.24$ & $<0.001$ & $0.95 \pm 0.49$ & $2.09 \pm 0.61$ & $<0.001$ \\
\hline
\end{tabular}

Values are presented as mean $\pm \mathrm{SD}$.
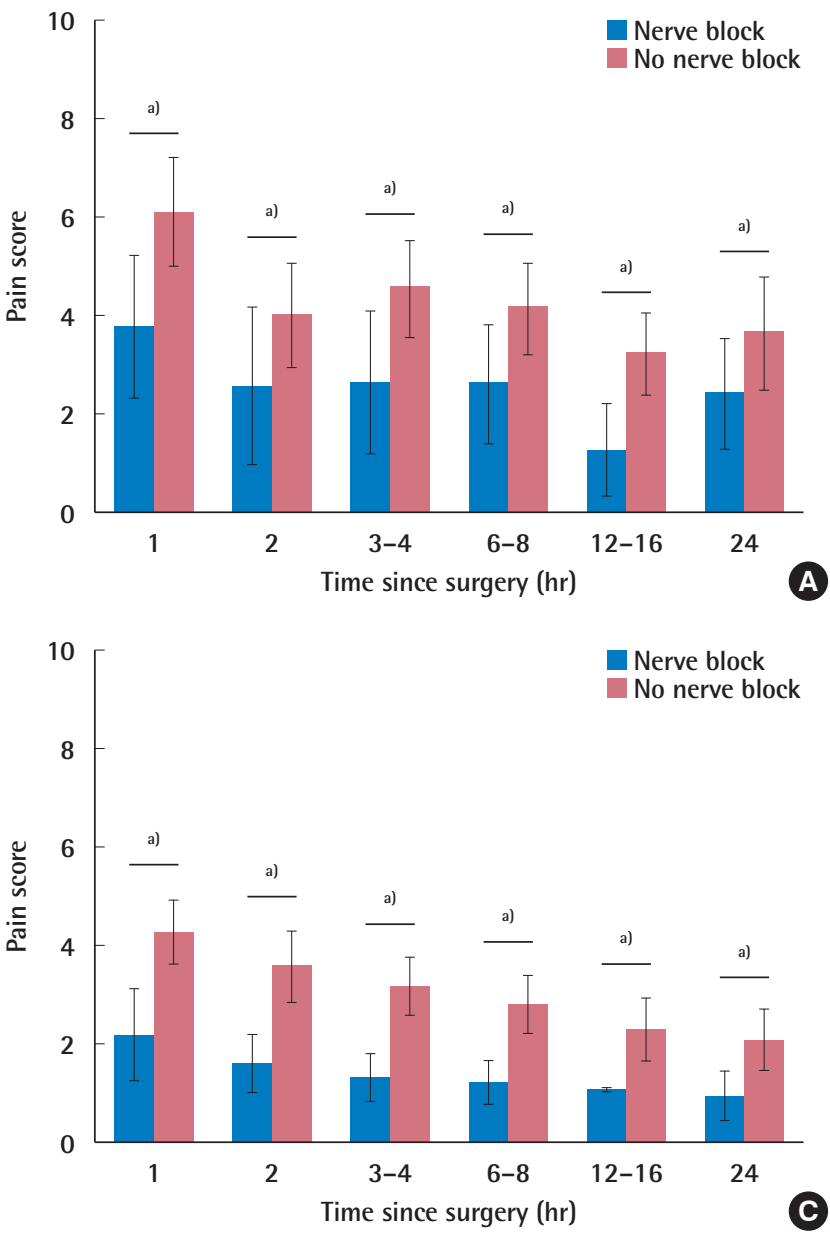

block group also averaged less pain at each time interval recorded, indicating that a block was correlated with less pain throughout the postoperative period (Fig. 1A). In terms of subgroup

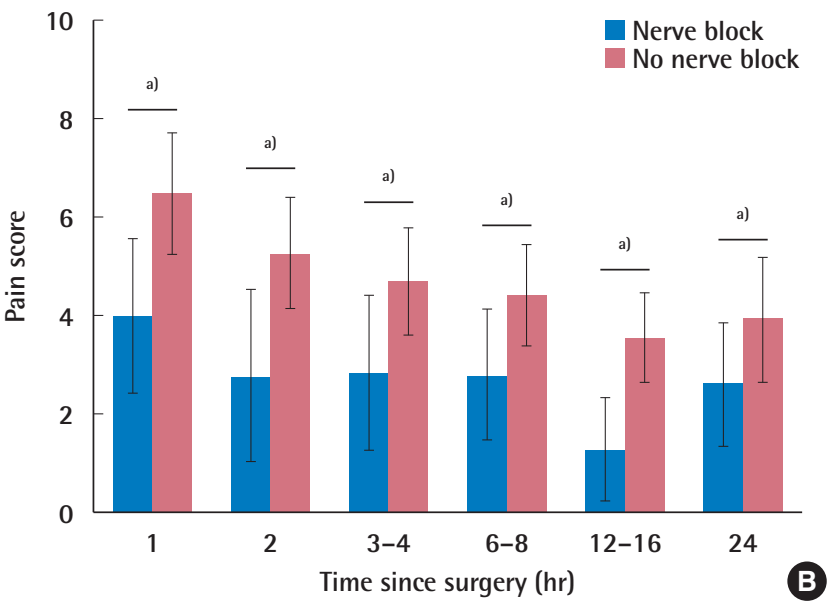

Fig. 1. Comparison of pain scores at all time intervals between nerve block and non-nerve block groups for (A) all studies, (B) breast augmentation cases, and $(C)$ reduction mammoplasty cases. ${ }^{\text {a) }} \mathrm{P}<0.001$.

analysis, this additionally held true when comparing augmentation procedures with and without blocks (Fig. 1B), and reduction procedures with and without blocks (Fig. 1C). Looking 


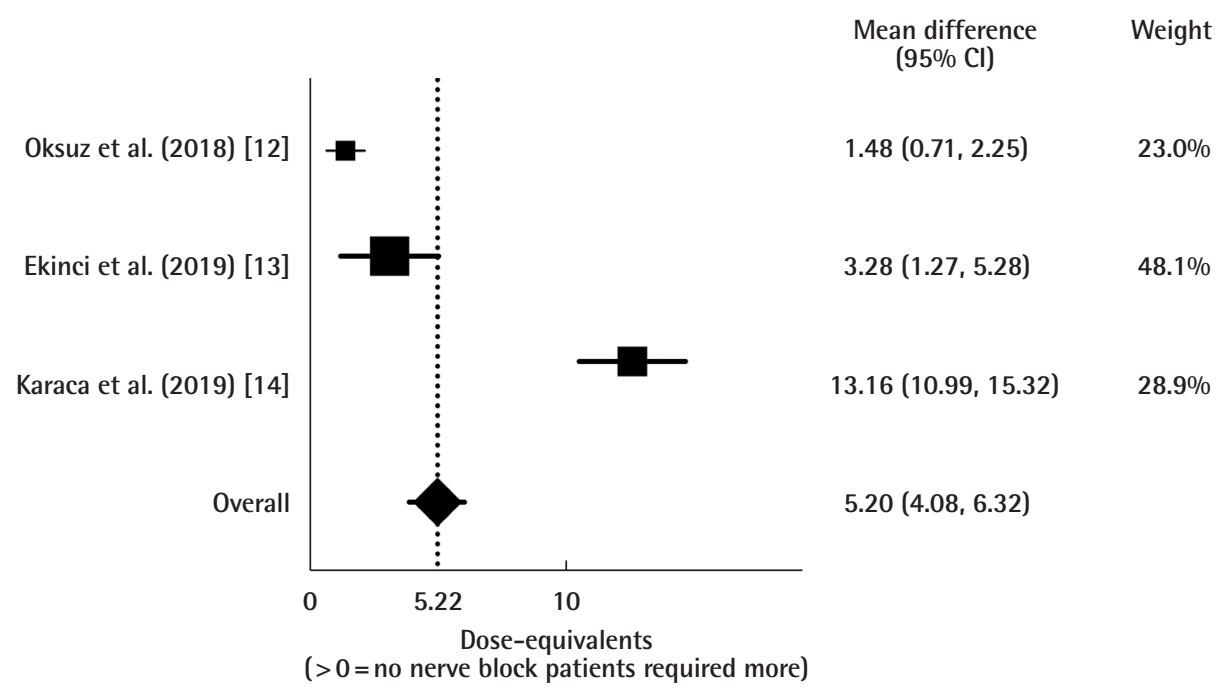

Fig. 2. Forest plot detailing differences in dose-equivalents of opioids consumed between nerve block and non-nerve block groups.

solely at subpectoral augmentation, 24-hour pain scores were $1.16,0.07$, and 2.9 for type-I, type-II, and type-II with serratus plane blocks, respectively $(\mathrm{P}<0.001$ for the overall and all pairwise comparisons).

Combining all studies, the nerve block group averaged $7.25 \pm 4.62$ dose-equivalents of opioids consumed in total, whereas the non-nerve block group averaged $12.45 \pm 2.35$ $(\mathrm{P}<0.001)$. This equated to a mean difference of $5.20 \pm 0.57$ (95\% confidence interval, 4.08-6.32) dose-equivalents favoring the non-nerve block group, indicating that a block correlated with less narcotic use (Fig. 2).

\section{DISCUSSION}

Body contouring operations are associated with considerable postoperative pain. Opioids are necessary to make these patients comfortable in the time periods following their procedures. These medications are equally as important for earlier mobilization, shortened hospital stays, reduced hospital costs, and increased patient satisfaction [16]. Nevertheless, a fine balance must exist between expectation of narcotic distribution by patients and reality of distribution by plastic surgeons.

We have found that nerve blocks not only reduce postoperative pain, but also the amount of opioids consumed per elective breast surgery patient. At all time intervals, from shortly after the surgery's end to a full day later, patients who received a block rated their pain significantly lower on standardized 0-10 scales ( 2.40 vs. 3.64 after 24 hours). This was further observed when stratifying by type of surgery, either breast augmentation or reduction mammoplasty. Furthermore, those who were blocked average more than 5 dose-equivalents of opioids less to manage pain. Nerve blocks have previously been investigated to be safe alternatives in plastic surgery $[17,18]$, as have patient-controlled pain pumps [19], which makes our study substantiate the utility of auxiliary interventions. Taking into account only subpectoral augmentation, known to be extremely painful postoperatively, type-II blocks resulted in the least pain and type-II with serratus plane blocks resulted in the most, with type-I blocks in the middle. This suggests that type-II blocks are most beneficial for reducing pain after subpectoral augmentation, but a prospective randomized clinical trial would be needed to strengthen the suggestion.

The present study did come with its limitations. No two studies were completely alike in analgesic intervention, thereby preventing a more robust analysis of a certain nerve block technique. Inconsistency of time points between studies precluded generation of a random effects model for pain scores. Lastly, follow-up time to record the number of opioids consumed was not standardized, ranging from a few days to a couple of weeks, which undoubtedly caused some sampling error.

As institutions continue to crack down on narcotic consumption as per regulations by the government, we anticipate the establishment of more stringent and even universal protocols. This should certainly be viewed as a positive for plastic surgery and the medical community as a whole. Regardless, it is most crucial to keep patient needs in mind so that the plastic surgeon can consistently provide comforting and healing experiences.

In conclusion, perioperative nerve blocks following body contouring of the breasts help patients with pain and the subsequent potential for opioid overdose. This is especially true for individuals undergoing subpectoral augmentation, after which pain is notoriously significant. 


\section{NOTES}

\section{Conflict of interest}

No potential conflict of interest relevant to this article was reported.

\section{Author contribution}

Conceptualization: DB Asserson, DE Sahar. Data curation, formal analysis: DB Asserson. Methodology, project administration, visualization: DB Asserson, DE Sahar. Writing - original draft: DB Asserson. Writing - review \& editing: DB Asserson, DE Sahar.

\section{ORCID}

Derek B. Asserson https://orcid.org/0000-0002-5505-7798

\section{REFERENCES}

1. Centers for Disease Control and Prevention (CDC). Understanding the epidemic [Internet]. Atlanta, GA: CDC; c2019 [cited 2019 Jul 13]. Available from: https://www.cdc. gov/drugoverdose/epidemic/index.html.

2. Waljee JF, Li L, Brummett CM, et al. Iatrogenic opioid dependence in the United States: are surgeons the gatekeepers? Ann Surg 2017;265:728-30.

3. Bicket MC, Long JJ, Pronovost PJ, et al. Prescription opioid analgesics commonly unused after surgery: a systematic review. JAMA Surg 2017;152:1066-71.

4. Ko JY, Tong VT, Haight SC, et al. Obstetrician-gynecologists' practice patterns related to opioid use during pregnancy and postpartum: United States, 2017. J Perinatol 2020; 40:412-21.

5. Prince LY, Mears SC, Watson JC, et al. Health literacy evaluation of opioid patient education materials for orthopaedic surgery.J Surg Orthop Adv 2019;28:232-36.

6. Boyd C, Shew M, Penn J, et al. Postoperative opioid use and pain management following otologic and neurotologic surgery. Ann Otol Rhinol Laryngol 2020;129:175-80.

7. Rose KR, Christie BM, Block LM, et al. Opioid prescribing and consumption patterns following outpatient plastic surgery procedures. Plast Reconstr Surg 2019;143:929-38.

8. Cooter RD, Rudkin GE, Gardiner SE. Day case breast augmentation under paravertebral blockade: a prospective study of 100 consecutive patients. Aesthetic Plast Surg 2007; 31:666-73.
9. Wallace MS, Wallace AM, Lee J, et al. Pain after breast surgery: a survey of 282 women. Pain 1996;66:195-205.

10. Ma J, Liu W, Hunter A, et al. Performing meta-analysis with incomplete statistical information in clinical trials. BMC Med Res Methodol 2008;8:56.

11. Hidalgo DA, Pusic AL. The role of methocarbamol and intercostal nerve blocks for pain management in breast augmentation. Aesthet Surg J 2005;25:571-5.

12. Oksuz G, Bilgen F, Arslan M, et al. Ultrasound-guided bilateral erector spinae block versus tumescent anesthesia for postoperative analgesia in patients undergoing reduction mammoplasty: a randomized controlled study. Aesthetic Plast Surg 2019;43:291-6.

13. Ekinci M, Ciftci B, Celik EC, et al. The efficacy of different volumes on ultrasound-guided type-I pectoral nerve block for postoperative analgesia after subpectoral breast augmentation: a prospective, randomized, controlled study. Aesthetic Plast Surg 2019;43:297-304.

14. Karaca O, Pinar HU, Arpaci E, et al. The efficacy of ultrasound-guided type-I and type-II pectoral nerve blocks for postoperative analgesia after breast augmentation: a prospective, randomised study. Anaesth Crit Care Pain Med 2019; 38:47-52.

15. Schuitemaker RJB, Sala-Blanch X, Sanchez Cohen AP, et al. Analgesic efficacy of modified pectoral block plus serratus plane block in breast augmentation surgery: a randomised, controlled, triple-blind clinical trial. Rev Esp Anestesiol Reanim (Engl Ed) 2019;66:62-71.

16. Morales RJr, Mentz H 3rd, Newall G, et al. Use of abdominal field block injections with liposomal bupivicaine to control postoperative pain after abdominoplasty. Aesthet Surg J 2013;33:1148-53.

17. Kennedy GT, Hill CM, Huang Y, et al. Enhanced recovery after surgery (ERAS) protocol reduces perioperative narcotic requirement and length of stay in patients undergoing mastectomy with implant- based reconstruction. Am J Surg 2020;220:147-52.

18. O’Neill RC, Hayes KD, Davison SP. Safety of postoperative opioid alternatives in plastic surgery: a systematic review. Plast Reconstr Surg 2019;144:991-9.

19. Chavez-Abraham V, Barr JS, Zwiebel PC. The efficacy of a lidocaine-infused pain pump for postoperative analgesia following elective augmentation mammaplasty or abdominoplasty. Aesthetic Plast Surg 2011;35:463-9. 